\title{
Experiencias de las adolescentes que cursan un embarazo, desde un enfoque cualitativo
}

\author{
Silvia Vianey Reyes-López ${ }^{1}$ (D); Iran Carolina Cordero-Cruz 2 (D); Francisco Javier Báez-Hernández ${ }^{3}$ (D); \\ Vianet Nava-Navarro ${ }^{4}$
}

\begin{abstract}
RESUMEN
Introducción: En los últimos años el embarazo en las adolescentes se ha convertido en un alarmante problema de salud pública ya que cada año 16 millones de adolescentes entre 15 y 19 años se convierten en madres. Objetivo: Describir las experiencias de las adolescentes que cursan un embarazo, desde un enfoque cualitativo. Metodología: estudio cualitativo, descriptivo, en el que participaron nueve adolescentes embarazadas, las cuales fueron seleccionadas con muestreo por conveniencia. Se realizaron entrevistas semiestructuradas hasta la saturación de datos; la investigación se apegó a la Ley General de Salud, en materia de investigación. Resultados: Los hallazgos de los discursos de las adolescentes se estructuraron en torno a tres categorías principales: 1) factores que contribuyen al embarazo con cuatro subcategorías: mal uso de métodos anticonceptivos, cohibición para preguntar sobre métodos anticonceptivos, relaciones sexuales no planeadas y consumo de sustancias; 2) reacción ante la noticia de embarazo con dos subcategorías: reacción positiva y adversa y 3) cambios ante el embarazo, con tres subcategorías: cambios en la escuela, cambios físicos y fisiológicos y cambios en el comportamiento social. Conclusiones: Las experiencias de las adolescentes acerca de su embarazo abarcan una gama de situaciones ambivalentes. Las experiencias que enfrenta una adolescente embarazada son multidimensionales; se requiere abordaje integral y multidisciplinario.
\end{abstract}

Palabras clave: Experiencias; Embarazo en Adolescencia; Investigación Cualitativa (DeCs).

\footnotetext{
${ }^{1}$ Licenciada en Enfermería. Facultad de Enfermería de la Benemérita Universidad Autónoma de Puebla, México. ${ }^{2}$ Licenciada en Enfermería. Facultad de Enfermería de la Benemérita Universidad Autónoma de Puebla, México.

${ }^{3}$ Doctor en Ciencias de Enfermería. Facultad de Enfermería de la Benemérita Universidad Autónoma de Puebla, México.

${ }^{4}$ Doctora en Ciencias de Enfermería. Facultad de Enfermería de la Benemérita Universidad Autónoma de Puebla. Puebla, México.
}

*Autor para correspondencia: vianet.nava@correo.buap.mx

Recibido: $26 / 01 / 2020$

Aceptado: $13 / 08 / 2020$

Cómo citar este artículo

Reyes-López SV, Cordero-Cruz IC, Báez-Hernández FJ, Nava-Navarro V. Experiencias de las adolescentes que cursan un embarazo, desde un enfoque cualitativo. SANUS. 2020; (15): 1-14. [Acceso dd $\underline{\mathrm{mm}} \underline{\text { aal }}$ : Disponible en: URL/DOI. 


\title{
Qualitative approach to experiences of pregnant adolescents
}

\begin{abstract}
Introduction: In recent years, adolescent pregnancy has become an alarming public health problem since each year 16 million adolescents between 15 and 19 years of age become mothers. Objective: Describe experiences of pregnant teenagers, from a qualitative approach. Methodology: Qualitative, descriptive study, where nine pregnant adolescents participated, who were selected using convenience sampling. Semi-structured interviews were carried out until data saturation; the research adhered to the General Health Act for research. Results: The findings of the discourse of the adolescents were structured around three main categories: 1) factors that contribute to pregnancy with four subcategories: misuse of contraceptive methods, self-restraint to ask about contraceptive methods, unplanned sexual relations and consumption of substances; 2 ) reaction to the news of pregnancy with two subcategories: positive and adverse reaction; and 3) changes to pregnancy, with three subcategories: changes in school, physical and physiological changes, and changes in social behavior. Conclusions: The experiences of adolescents with respect to their pregnancy cover a range of ambivalent situations. Additionally, the experiences that a pregnant adolescent faces are multidimensional; thus, a comprehensive and multidisciplinary approach is required.
\end{abstract}

Keywords: Experiences; Pregnancy in Adolescence; Qualitative Research (DeCS). 


\title{
Experiências de adolescentes grávidas de uma abordagem qualitativa
}

\begin{abstract}
ABSTRATO
Introdução: Nos últimos anos, a gravidez na adolescência se tornou um alarmante problema de saúde pública, uma vez que 16 milhões de adolescentes entre 15 e 19 anos se tornam mães a cada ano. Objetivo: Descrever as experiências das adolescentes que estão grávidas a partir de uma abordagem qualitativa. Metodologia: estudo abordagem qualitativa, descritivo, participaram nove adolescentes gestantes, selecionadas com amostragem por conveniência. As entrevistas semiestruturadas foram realizadas em profundidade até a saturação dos dados; a investigação aderiu à Lei Geral de Saúde, em termos de pesquisa. Resultados: Os achados dos discursos de adolescentes foram estruturados em torno de três categorias principais: 1 ) fatores que contribuem para a gravidez em quatro subcategorias: uso indevido de métodos contraceptivos, inibição de perguntar sobre métodos contraceptivos, relações sexuais não planejadas e consumo de substâncias; 2) reação às notícias da gravidez com duas subcategorias: reação positiva e reação negativa e 3) mudanças antes da gravidez, com três subcategorias: mudanças na escola, mudanças físicas e fisiológicas e mudanças no comportamento social. Conclusões: As informações obtidas mostram que as experiências das adolescentes sobre a gravidez abrangem uma gama de atitudes ambivalentes que podem ter um relacionamento complexo. As experiências enfrentadas por uma adolescente grávida são complexas e causas multifatoriais que requerem atenção especial por sua abordagem abrangente e multidisciplinar.
\end{abstract}

Palavras-chave: Experiências; Gravidez na adolescência; Pesquisa qualitativa (DeCS). 


\section{INTRODUCCIÓN}

En los últimos años el embarazo en las adolescentes se ha convertido en un alarmante problema de salud pública ya que cada año aproximadamente 16 millones de adolescentes entre 15 y 19 años se convierten en madres, de las cuales 1 millón son menores de 15 años; la mayoría provenientes de países en vías de desarrollo(1). En América Latina, la incidencia de embarazo en adolescentes es de 46 nacimientos por cada 1,000 (2). Así mismo, México se encuentra en primer lugar de embarazo en adolescentes, por cada 1,000 mujeres de 15 a 19 años existen 77 nacimientos, lo que significa que aproximadamente el $31.2 \%$ de los adolescentes han comenzado a tener vida sexual activa sin uso de métodos anticonceptivos ${ }^{(3)}$. El estado de Puebla, se ubicó en el octavo lugar a nivel nacional con el mayor número de embarazos adolescentes al registrar que 25 mil 608 mujeres menores de 20 años de edad tuvieron un parto durante el $2017^{(4,5)}$.

Ante este panorama, es necesario precisar que la adolescencia es el periodo de los 10 a 19 años, en el que se presentan transiciones biológicas, psicológicas, sociales y económicas ${ }^{(6)}$, etapa en la que, al presentarse un embarazo, se inicia una serie de condiciones de vulnerabilidad que afectan la salud, educación y proyecto de vida de la adolescente, asociadas a la falta de preparación para esta nueva responsabilidad $^{(7)}$. Por lo que viven las condiciones de la maternidad no planeada, lo cual es representado por ser frecuentemente estigmatizadas, sufrir discriminación de género, desaprobación y rechazo familiar, además de la indiferencia de la sociedad; lo cual desencadena en ellas una serie de frustraciones sociales $y$ personales ${ }^{(8,9)}$.

De esta forma, el embarazo a temprana edad se convierte en un problema multifactorial que genera causas individuales, familiares, socioculturales y políticas ${ }^{(10)}$; entre las cuales se encuentra el inicio de vida sexual activa precoz ${ }^{(11)}$, maduración sexual temprana ${ }^{(12)}$, bajo nivel educativo ${ }^{(13)}$, falta de planificación familiar, educación sexual ineficaz ante la falta de comunicación con los padres respecto a temas de sexualidad $y$, a su vez, la familia posee poco conocimiento sobre educación sexual ${ }^{(9)}$. Entre los factores socioculturales que se enmarcan son el estrés, la delincuencia, las adicciones, las creencias, mitos y tabúes sexuales, marginación social y predominio del amor romántico en las relaciones $(14,15)$.

Por lo anterior, el profesional de enfermería tiene el compromiso de profundizar sobre este fenómeno con el fin de generar un cambio en el paradigma con el que se aborda actualmente esta compleja situación; así mismo, es necesario el acercamiento a las experiencias de las adolescentes embarazadas, a partir de la teoría de las representaciones sociales ${ }^{(16)}$, lo que permite comprender de manera flexible la posición intermedia entre el significado que se obtiene del sentido real, y la imagen que las adolescentes reelaboran de las condiciones individuales, familiares y socioculturales, que parten de un conjunto de ideas, saberes y conocimientos para actuar e interpretar su realidad inmediata.

Las representaciones propuestas por Moscovici ${ }^{(16)}$, se estructuran alrededor de tres componentes fundamentales: la información que se posee, el campo de representación donde se organizan jerárquicamente una serie de contenidos y la actitud hacia la situación, con base en esta propuesta, es posible profundizar en las experiencias de las adolescentes en su diálogo nos permiten comprender su proceso de aceptación a una nueva realidad de su vida, lo que permitirá brindar elementos que consoliden una atención para la prevención y atención del embarazo en las adolescentes por medio de intervenciones de enfermería oportunas y programas educativos que fortalezcan las debilidades en salud de los adolescentes adecuándolas a su contexto y realidad. Por lo anterior, el objetivo de este estudio es describir las experiencias de las adolescentes que cursan un embarazo, desde un enfoque cualitativo. 


\section{METODOLOGÍA}

Se trata de un estudio de tipo cualitativo(17-18), descriptivo ${ }^{(19)}$ debido a que permitió describir las experiencias de las adolescentes que cursan un embarazo; la selección de las participantes fue a través de un muestreo por conveniencia, con un total de nueve adolescentes por disponibilidad y hasta lograr la saturación de los datos; los criterios de inclusión fueron ser adolescentes embarazadas entre los 15 a 19 años, que recibieran atención de salud en una Unidad Médico Familiar (UMF) de una Institución de Salud Púbica de la Ciudad de Puebla, que contarán con el consentimiento firmado de la madre/padre o tutor, asentimiento informado y que aceptarán participar en la investigación; se consideró como criterio de eliminación a las adolescentes que estuvieran embarazadas producto de violación sexual. Los investigadores desempeñaron un rol como observador no participante, al cumplir la función como espectador en el desarrollo de las entrevistas semiestructuradas( ${ }^{(20)}$, las cuales se realizaron a través de una guía de preguntas, con el fin de comprobar su estructura y comprensión se realizó una prueba piloto (21). La entrevista estuvo conformada por preguntas introductorias, cuya finalidad fue establecer la confianza entre la participante y el investigador, las siguientes preguntas se estructuraron a partir de la revisión de literatura y el resto haciendo hincapié sobre las experiencias del embarazo en las adolescentes que contribuyen en la profundización de este fenómeno de estudio, las reacciones frente a la noticia de embarazo y los cambios del mismo. Una serie de preguntas previamente definidas y abiertas, con la libertad de introducir preguntas adicionales para precisar conceptos $\mathrm{u}$ obtener mayor información ${ }^{(19)}$. Las entrevistas permitieron una comunicación libre, espontánea y sin interrupciones, así como la construcción conjunta de significados respecto a esta temática. Cada una de las entrevistas tuvo una duración aproximada de una hora y se realizaron en un ambiente tranquilo y privado, lo que les generó un ambiente de intimidad y confianza, para poder responder de forma tranquila. Dichas entrevistas fueron grabadas en audio y posteriormente transcritas en el programa de Microsoft Office Word 2010.

El análisis de datos fue a través de lo propuesto por de Braun y Clarke ${ }^{(22)}$, el cual consiste en seis fases: 1) familiarización de los datos, en donde se transcribieron las nueve entrevistas audio grabadas anotando ideas iniciales mediante mapas mentales; 2 ) se realizó la generación de códigos en el programa MAXQDA versión 18.2.0, ubicando características interesantes y cotejando datos relevantes; efectuando un reporte de códigos vivos para ubicarlos por campos semánticos; 3 ) se buscaron temas sobre los factores que contribuyen al embarazo, reacción ante la noticia de embarazo y cambios ante el embarazo; 4) para cotejar los códigos en temas potenciales se hizo una revisión de las categorías y subcategorías para comprobar que los temas funcionaran en relación a los códigos, lo que permitió la realización de un mapa temático, generando una definición de cada categoría; 5) definición y denominación de temas, en donde se establecieron jerarquías (temas y subtemas) que permitieron el análisis a partir de las dimensiones de la teoría de las representaciones sociales ${ }^{(16)}$; finalmente 6 ) se realizó el informe de la investigación, sustentado en la comprensión e interpretación de la información obtenida (22).

La investigación se sometió para su aprobación al Comité de Investigación y Estudios de Posgrado de la Facultad de Enfermería de la Benemérita Universidad Autónoma de Puebla con el registro SIEP/032/2020, así mismo, se rigió con base al Reglamento de la Ley General de Salud ${ }^{(23)}$ en materia de investigación donde se establece la participación de forma voluntaria, los principios de confidencialidad y el requerimiento de un consentimiento y un asentimiento informado debidamente firmado; además, de tomarse en cuenta los lineamientos para la investigación en menores de edad y grupo de embarazadas.

Por cuestiones de ética, se apegó al principio de confidencialidad toda la información que precisara la identidad de las participantes, las cual fueron protegidas bajo seudónimos y códigos $^{(19)}$. Los criterios de calidad considerados fueron: credibilidad, al realizar la 
transcripción textual del total de entrevistas semiestructuradas; consistencia para el análisis de datos y confirmabilidad, debido a que la información que se recabó fue de manera inductiva ${ }^{(20)}$.

Se entrevistaron a nueve adolescentes que cursan un embarazo, cinco de ellas tienen 17 años, tres 16 años y una con 18 años. En relación con su estado civil cuatro se encuentran en unión libre, dos permanecen solteras y tres se encuentran casadas, en cuanto al análisis de contenido a partir de las dimensiones de la teoría de las representaciones sociales de Moscovici ${ }^{(16)}$, se identificaron tres categorías y nueve subcategorías. La primera categoría

\section{RESULTADOS}

corresponde a los factores que originan el embarazo, se ubica en la dimensión de información. La segunda categoría se refiere a los cambios en el embarazo y corresponde a la dimensión del campo de representación; y la tercera categoría es reacción ante el embarazo a través de la dimensión de actitud (Figura 1).

Figura 1. Experiencias de las adolescentes de 15 a 19 años que cursa un embarazo.

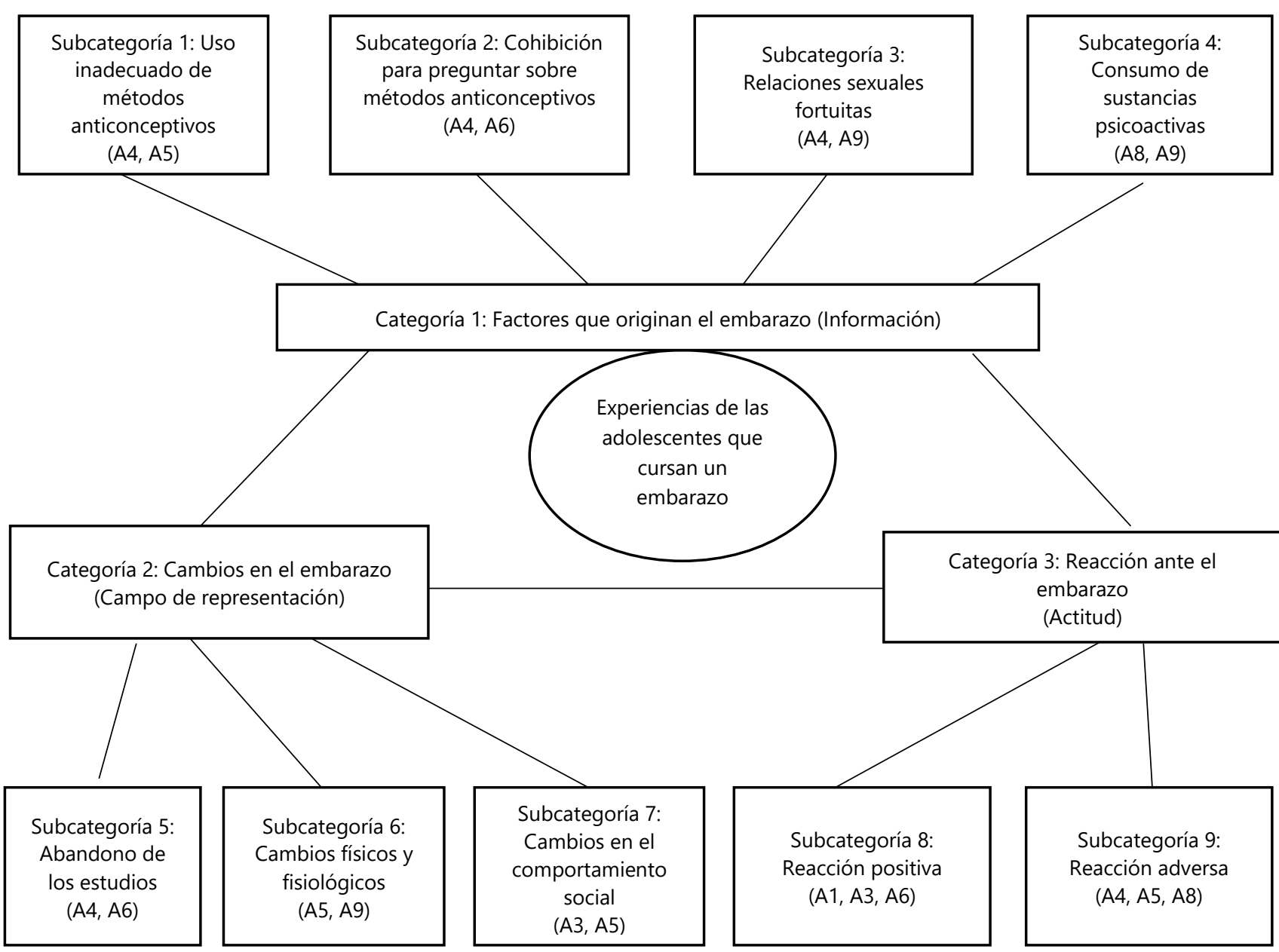

Fuente: Elaboración propia 
De acuerdo a lo propuesto por Moscovici(16), las representaciones sociales emergen de las condiciones construidas en momentos de crisis, en el abordaje de este estudio corresponde al embarazo que cursa la adolescente, por lo que en la categoría 1: factores que originan el embarazo, se presenta una condición de emergencia, en la cual, la información se encuentra dispersa y no es suficiente, por lo regular esta desorganizada, y es como se expresa en el discurso de las adolescentes respecto al uso inadecuado de los métodos anticonceptivos (subcategoría 1), las participantes mencionan que han escuchado sobre los métodos anticonceptivos, sin embargo, no saben cómo utilizarlos, consideran que la pastilla de emergencia puede utilizarse como método anticonceptivo: "sí, sí sabía que existían, porque cuando platicábamos con mis amigas luego platicaban que había estado con sus novios y que se ponían condón [...] pues si, como que, pues si me lo trataba de imaginar, pero no [...] este, nunca les preguntaba bien cómo es que se ponía [...]" A4, 16 años; "iyo siempre que tenía relaciones utilizaba las pastillas del día siguiente, iósea!, porque no me gustaba usar el condón [...] pero algunas veces cuando estábamos juntos a veces se le salía y pues no servía, iy terminaba quitándoselo![...] y como no teníamos relaciones tan seguido pues yo me tomaba pastillas[...] pero aaaah! Pues sí, si sabíamos que existían los métodos anticonceptivos" A5, 17 años.

Otro elemento es que la fuente de información en relación a la cantidad y calidad de acuerdo a los campos de interés, vuelven precarios los vínculos con el juicio y puede ser complejo buscar la información y operacionarla en la cotidianidad $^{16}$. Expresando cohibición para preguntar sobre métodos anticonceptivos (subcategoría 2), no preguntan debido a que sienten pena: "Pues la verdad solo veía a veces como se lo ponía mi novio este, es que no sabes (sonríe) la verdad me daba pena porque como les iba a preguntar a mi familia o a mis amigas" A4, 16 años.

La información de métodos anticonceptivos se encuentra al alcance de las adolescentes, sin embargo las adolescentes no expresan sus inquietudes sobre temas sexuales con la familia: "Es que la verdad si habían hablado de eso en la escuela pero pues por pena o no sé yo o porque, prefieres echar relajo o no hacer caso y pues la verdad no ponía atención cuando nos explicaban y pues si me daba pena hablarlo con mis papás o con alguien ya que no quería que pensaran mal de mí, pues era hija de casa y pues eso no estaba bien, ya sabes" A6, 16 años.

La información y experiencia que posee la adolescente en relación a posibles escenarios en los cuales pueden ser de riesgo para para tener relaciones sexuales fortuitas (subcategoría 3), como acontecimientos de la cotidianidad, donde el discurso resalta relaciones sexuales sin el uso del condón, dejándose llevar por el momento: "Puesss jaaaah! ¡No sé por qué no se cuida uno! [...] o iporque piensa uno que es fácil tener relaciones sin condón!, bueno en realidad no sé por qué, pero sí creo que a veces te dejas llevar por el momento y ino piensas lo que puede pasar! " A5, 17 años. Así mismo, las participantes mencionan que el tiempo del noviazgo fue corto cuando deciden iniciar sus relaciones sexuales, aunado al uso inadecuado de métodos anticonceptivos, surge el embarazo: "después de un ratito de novios empezamos a tener relaciones y la verdad en el momento en que me embarace no nos cuidamos muy bien que digamos y pues ese día menos pensado, fuimos a una casa con [...] bueno con unos amigos, después él después me fue a dejar a mi casa y pues jehm! ahí se dio la oportunidad (sonrojada) por que no estaba nadie" A9, 17 años.

Así mismo, después del consumo de sustancias (subcategoría 4) como alcohol o drogas tienen relaciones sexuales, limitadas en la toma de decisión y uso de protección: "fue por el momento, pues en la fiesta también habíamos tomado un poco de alcohol y pues ya a lo mejor con alcohol [...] iuno se arma de valor! y pues no piensa las cosas como deben de ser, ni él ni yo pensamos en cuidarnos [...] ni en algo, en ir a comprar algo o que si ya no nos habíamos cuidado en ese momento a lo mejor al día 
siguiente conseguir algún medicamento o algo para evitar un embarazo" A9, 17 años.

Otra adolescente menciona de igual manera que al consumir droga no se acordaba si había tenido relaciones sexuales: "Todos empezamos a tomar y unos empezaron también como a drogarse y pues esa vez no me obligó a probarla pero si me dijo que iba a ser una experiencia nueva [...] fuimos a su casa a seguir platicando como no estaba su tía nos pudimos subir a su cuarto y pues ya ipaso lo que paso!, no fue mi primera vez, pero la verdad ni me acuerdo bien como fue y ya después me quede como dormida y pues me despertó y ya me dijo que me fuera porque él iba a volver a salir y ya me acerco a mi casa [...] mi madrina me convenció para sacarme sangre de mi brazo fue cuando ya vino su compañera con los resultados y me dijo iAy, chamaca! ¡Ya saliste con tu premio!" A8, 16 años. Con base en la categoría 2, que corresponde a los cambios en el embarazo, ubicada de acuerdo al marco interpretativo en la dimensión del campo de representación, las adolescentes implicadas en la interacción social, focalizan hechos que evocan al juicio desde una mirada única para la toma de decisiones, por lo que uno de los cambios que se presenta es el abandono de los estudios (subcategoría 5), debido a la incomodidad de ser observadas, señaladas y estigmatizadas "cuando yo voy a la escuela desde que se enteraron que estoy embarazada, pues ya no es lo mismo, porque ya toda la escuela se dio cuenta y entonces siento que cuando salgo a comprar o voy a algún lado me ven raro [...]. Nada más se me quedan viendo [...]. $Y$ luego cuando voy caminando, escucho que hablan cosas [...] o empiezan a decirse cosas [...], se cuchichean de que yo voy pasando, los maestros también me ven como que se hacen que no pasa nada, pero yo siento que se me quedan viendo" $\mathrm{A} 4,16$ años. "Pues la verdad si intente ir a la escuela un rato, pero si me sentía incomoda por las náuseas y me daba miedo que me pasara algo y pues aparte la verdad no me gustaba que me vieran raro los demás alumnos isi era muy molesto que se me quedaran viendo o hablaran de mí!" A6, 16 años.

Las adolescentes mencionan que los cambios físicos y fisiológicos (subcategoría 6) como aumento de peso, sueño, fatiga, náuseas, incertidumbre e incomodidad limitan sus actividades

"iPues ahora ya me empecé a vestir más aguado porque pues ya no me queda mi ropa de antes, ya me puse más [...] ósea más llenita! ilgual las bubis me están creciendo! ¡No sé [...] aaaah! No sé qué vaya a pasar después de tener al bebé" A5, 17 años. "Y pues, ya no me siento igual con los cambios en el embarazo, tengo náuseas o me duele la cabeza o me da muchísimo sueño, me duele la cintura, me canso mucho cuando camino" A9, 17 años.

Las adolescentes refieren que a partir del embarazo, se enfrentaron a cambios en el comportamiento social (subcategoría 7), entre estos, desempeñar otros roles frente a la sociedad, deben atender su embarazo y a su pareja; añoran cuando podían salir a divertirse con sus amigas: "Ahora yo, tengo que hacer todo, pues si, fue un cambio, como que iay!, pues si, en mi forma de ser, pues que ya no eres una niña, ya tienes que comportarte como una [...], como una mujer, iComo una señora!" A3, 17 años. "iSi, ya cambié porque antes salía mucho hacia desmadre [...] era mucho de echar relajo y pues era más divertida! Ahora como que ya me volví más tranquila, ya también porque como estoy en la casa de mis suegros pues [...] ya, ni modo que me salga a echar relajo [...] pues no, que van a decir, y pues si así apenas me quieren ¡Imagínate!" A5, 17 años.

Para la categoría 3: Reacción ante el embarazo, la dimensión de actitud se refiere a la orientación favorable o desfavorable en relación a la condición que esto implica, el cual es un componente hacia la motivación al tomar posición respecto a la experiencia que esta representación social significa para la adolescente en su sistema de valores, ideas y prácticas. Se encontró una reacción positiva (subcategoría 8) algunas participantes expresan sentir felicidad al enterarse de la noticia de embrazo, particularmente las que tienen una pareja y familia que las apoya, además refieren con inquietud los cambios que surgirían: "Me sentí ¡muy feliz! por que como tenía problemas hormonales me habian dicho que probablemente no me iba a poder embarazar tan fácil" A1, 17 años. 
Una situación sobre el embarazo, es informarle a las parejas de las adolescentes, los cuales, de acuerdo al discurso de las participantes, son los primeros en saber sobre el embarazo y como parte de este proceso de aceptación, en el caso de las adolescentes con pareja, las apoya: "Él primero que se enteró fue mi pareja y immm? pues igual no, no se lo esperaba, pero pues, si me apoyó" A3, 17 años. "Al principio si se alteró, $y$ me dijo que como era posible que eso no lo teníamos planeado, pero pues creo que como me vio que solo estaba llorando pues si me abrazo y se tranquilizó, dijo que él iba a hablar con mi familia y pues que sí que se haría responsable y que hablaría con sus papas para ver qué pasaría" A6, 16 años.

La red de apoyo familiar estuvo presente en el discurso de las participantes: "Sí nos poyaron, la familia de él, nos apoyaron, si nos regañaron un poco y ya después nos dijeron que teníamos su apoyo que contábamos con ellos y pues ya si fue un poco difícil" A3, 17 años. "Ya mis papás estaban medio inquietos porque si me vieron llorando y pues cuando les dije pues ifue como si explotara una bomba! porque pues como podía ser cierto que yo estuviera panzona y demás. $Y$ si me puse a llorar, pero pues al final mis papás me dijeron que me apoyarían y ya cuando estuvimos todos reunidos, $\mathrm{mmm}$, pues mis papás y sus papás hablaron y pues ya al final decidimos que queríamos casarnos y vivir juntos y pues sus papás nos dieron un cuarto para que estuviéramos y ya hiciéramos nuestras cosas y si pues sí, así la llevamos" A6, 16 años.

Las reacciones ante el embarazo en la adolescente fueron diversas, desde sorpresa, confusión, temor, incertidumbre, incluso al enfrentarlo con la pareja y familia; en la subcategoría 9, se identifica una reacción adversa, ya que la circunstancia ejerce presión en la respuesta de la adolescente frente a este contexto conflictivo: "En ese momento, sentía muchas cosas, no sé [...] icomo si me echaran una cubeta de agua fría! iy entonces, me puse a llorar, porque ¿cómo le iba a decir a mis papás que estaba embarazada?, en ese momento se me vinieron muchas cosas a la cabeza pensé que me iban a correr de la casa [...] ino inventes! jno te imaginas como me puse a llorar!" A5, 17 años. "Yo en ese momento quería desaparecer, la verdad estaba muy asustada porque no sabía que iba a pasar conmigo, luego pues yo pensé que mis papás me iban a correr de la casa porque como se enojaron [...]"A4, 16 años.

Dos de las adolescentes mencionaron, no recibir apoyo por parte de su pareja al momento de enterarse de la noticia: "Entonces me dijo que él iba a haber que hacía [...] pero después de una semana que le dije aún no me buscaba y ya no me buscó" A5, 17 años. "Pues el problema era ese güey, porque pues ya ni por face me contesto y pues en la calle lo veía y me ignoraba o me insultaba [...] con sus amigos se reían y demás y si sentífeo, pero ino le podía rogar que se hiciera responsable!, creo que ni si quiera me hubiera creído que era de él" A8, 16 años.

Una de las participantes refirió no contar con el apoyo de su familia "Sí, mi mamá se enojó, casi me corrió que porque hice eso que ella no quería que yo terminará, así como ella y ya me dijo que yo le tenía que buscar cómo debía de mantenernos y que pues yo me tenía que rascar con mis propias uñas porque a ella no le alcanzaba" A8, 16 años.

\section{DISCUSIÓN}

El objetivo de la presente investigación fue describir las experiencias de las adolescentes que cursan un embarazo desde un enfoque cualitativo respondiendo a las necesidades de manejo plasmadas en el tercer objetivo de desarrollo sostenible donde enfocan a las adolescentes como un grupo de riesgo frente al embarazo no deseado (24).

En cuanto a la categoría sobre los factores que originan el embarazo, coincide con lo propuesto por Chacón et al ${ }^{(9)}$, el cual menciona que la impulsividad ante el sexo, el uso de sustancias como el alcohol y el mal uso de los métodos anticonceptivos son factores de riesgo asociados al embarazo adolescente, lo que concuerda con los resultados encontrados en este estudio en donde la falta información e impulsividad ante el sexo es manejada como relaciones sexuales fortuitas debido a que las adolescentes mencionan encuentros sexuales inesperados, de la misma manera refieren haber consumido alcohol o drogas antes de la relación 
sexual, así como utilizar la pastilla de emergencia como método anticonceptivo habitual.

Mientras que los hallazgos relacionados con los sentimientos como la cohibición para preguntar que sienten las adolescentes al momento de despejar sus dudas sobre los métodos anticonceptivos coinciden con los resultados de Gómez y Durán ${ }^{(25)}$, donde ellos establecen que la edad de las adolescentes se vuelve una barrera de críticas y cuestionamientos que puede detener la búsqueda de los mismos.

En relación con los resultados obtenidos de las adolescentes con lo referente a mantener relaciones sexuales no planeadas se encuentra semejanza con lo plasmado en el trabajo de González y Quintana (26), donde se enmarca una clara tendencia a tener relaciones sexuales esporádicas y no planificadas marcadas por la impulsividad mostrándose como una de las características principales de las conductas sexuales propias de esa etapa.

En relación al consumo de sustancias psicoactivas, Carrillo y Manzanero ${ }^{(27)}$ mencionan que el consumo de alcohol se convierte en un riesgo que fomenta el embarazo no planeado en las adolescentes; coincidiendo con lo encontrado en las participantes. De igual forma Figueroa y Figueroa ${ }^{(14)}$, refieren que el consumo de esta sustancia disminuye la percepción de riesgo y cambia sus acciones, por lo que es más probable mantener relaciones sexuales sin protección, lo cual se plasma en el discurso de las adolescentes.

En la categoría sobre los cambios en el embarazo de las adolescentes que corresponde a la dimensión campo de representación, la subcategoría de abandono de los estudios, se muestra como un factor predominante, lo que coincide con la investigación de Mejía et al. (28) donde el dejar la escuela, se ve influenciado por la sintomatología clínica de la adolescente, las alteraciones en su imagen corporal y la imposibilidad de seguir realizando las actividades recreativas; de la misma manera Mora y Hernández ${ }^{(31)}$ coinciden con los resultados de este estudio, pues refiere que el estancamiento del desarrollo personal y abandono escolar son consecuencias del embarazo adolescente.
Con relación a esto, Quintero y Rojas ${ }^{(32),}$ mencionan que lo importante no es solo la presencia de la familia y la pareja sino el apoyo y la motivación para que las adolescentes embarazadas puedan continuar con sus estudios; hecho que evidentemente no se ve reflejado en nuestros resultados.

Se encontró que la futura madre se enfrenta a factores estresantes como cambios físicos, fisiológicos y psicológicos que modifican sustancialmente la vida de la adolescente por lo que tiene que adaptarse a estos nuevos cambios, esto coincide con lo que señala en su estudio Ramos y Borgues ${ }^{(33)}$ en donde mencionan que los cambios físicos en las adolescentes es un tema susceptible debido a que al percibir una imagen corporal diferente les genera inseguridad.

En la subcategoría de cambios en el comportamiento social se identificó que las adolescentes presentan cambios radicales debido a que adquieren nuevos roles sociales en los que ellas se ven obligadas a cambiar su comportamiento para asumir la maternidad y su responsabilidad, lo que concuerda con lo planteado por Niño et al ${ }^{(34)}$ en donde remarca que las adolescentes embarazadas alteran su red social de amigos y familia además de adquirir nuevos roles en la futura madre relacionados al hogar y la responsabilidad de formar una familia.

En referencia a la categoría de reacción ante el embarazo, que hacer referencia a la actitud, se encontraron reacciones ambivalentes tanto positivas como adversas; en cuanto a las reacciones positivas el código vivo encontrado por Mejía, et al.(28), refiere que la adolescente embarazada expresa lo siguiente: "estoy más feliz, mejoró mi relación con mi familia, me hizo madurar como persona y ya no estaré sola".

De igual forma Álvarez, et al. ${ }^{(15)}$, muestra en un código vivo del estudio realizado, con verbalizaciones positivas sobre la idea de estar embarazada: "Eso es un regalo muy lindo que nos da Dios [...], ¿Por qué uno le va a negar la vida a otra persona?"; estos códigos coinciden con los resultados encontrados en este estudio donde las adolescentes embarazadas mencionan reacciones positivas como: "Pues si me sentí feliz pero también a veces no sé, sentí 
que ya no iba a ser lo mismo que iba a tener cambios, muchos cambios" A2.

Las reacciones adversas que experimentan las adolescentes, tales como: "Me sentía a veces triste [...], algunos momentos yo decía que no quería tener el bebé" referido en el código vivo obtenido de Álvarez ${ }^{(15)}$, coinciden con los resultados de esta investigación en donde las adolescentes experimentan sentimientos tristeza y angustia.

Con relación al apoyo que reciben las adolescentes embarazadas por parte de la pareja y la familia, si bien en los resultados de este estudio se identificó apoyo por parte de la red familiar, se encontraron diferencias con lo que plantea Loredo, et al. ${ }^{(29)}$, mencionan que cuando la adolescente informa a la familia de su embarazo, habitualmente es reprendida y le generan culpa al hacerla responsable de su decepción e incluso en ocasiones es agredida emocional y/o físicamente.

Esta reacción probablemente varia por lo que Rojas, Méndez y Alvarez ${ }^{(30)}$, describen en su escrito, donde muestran que la percepción del embarazo de la adolescente dependerá del contexto social de los miembros de su familia. La descripción de las experiencias de las adolescentes embarazadas obtenidas a partir de un abordaje cualitativo y con el uso de un marco interpretativo de las representaciones sociales, permitió un acercamiento a este grupo vulnerable para la identificación de las dimensiones sobre información, actitudes, sentimientos, conocimientos, sucesos y del campo de representación que se hacen complejas durante la interacción con la adolescente. Es necesario que la enfermera oriente su mirada a la comprensión de los múltiples escenarios en los cuales ocurren las realidades, hacer que lo invisible resulte perceptible, lo que aporta a nuevas estrategias de cuidado.

En relación a las limitaciones del estudio, se consideró un diseño cualitativo, descriptivo porque el abordaje del fenómeno ha sido parcialmente abordado por enfermería, por lo que este diseño permite un acercamiento para explorar las experiencias de las adolescentes embarazadas. Otra limitación fue contactar a las participantes para el estudio, por ser menores de edad y por la condición de embarazo, es complicado el acercamiento a debido a situaciones sociales que se enfrentan en la actualidad, como es la inseguridad.

\section{CONCLUSIONES}

El embarazo adolescente es un proceso fisiológico en el cual se encuentran inmersos factores como el uso inadecuado o no uso de métodos anticonceptivos, la cohibición para preguntar sobre métodos anticonceptivos, así como las relaciones sexuales fortuitas contribuyen a que este problema quede aún sin resolver; donde la futura madre adolescente se enfrenta a cambios en la condición física, emocional y de comportamiento en la sociedad. Las experiencias que enfrenta una adolescente ante el proceso del embarazo son complejas y abarcan múltiples causas que requieren atención multidisciplinaria para su abordaje. Por lo que las acciones de los profesionales de enfermería juegan un papel fundamental en el cuidado de las adolescentes y en sus procesos de salud; así como en la prevención del embarazo a temprana edad, ya que permite detectar de manera oportuna y minimizar los factores de riesgo por medio de la atención de significados y experiencias de las adolescentes. Los resultados de este estudio permiten ser un punto de referencia al momento de plantear, diseñar y ejecutar acciones, estrategias y programas acordes a las necesidades de este grupo vulnerable ajustados a su realidad.

Finalmente, se plantea la necesidad de profundizar en el embarazo adolescente; con el fin de fomentar la realización de estudios con enfoque mixto en el ámbito de enfermería que permita abordajes no solo de exploración, si no que trascienda hacia enfoques multidisciplinarios, que ayuden a ofrecer una perspectiva más profunda para acrecentar el cuerpo de conocimientos que aporte al cuidado humanizado. 


\section{CONFLICTO DE INTERESES}

Se declara la inexistencia de un conflicto de intereses por parte de los autores de la investigación en relación con el presente artículo.

\section{FINANCIAMIENTO}

Esta investigación no fue financiada por ninguna institución.

\section{AGRADECIMIENTOS}

A todas las adolescentes que han participado en este estudio, al compartir sus experiencias.

\section{REFERENCIAS BIBLIOGRÁFICAS}

1. Organización Mundial de la Salud (OMS). Embarazo en la adolescencia [Internet]. OMS. 2018. [actualizado marzo 2020; consultado Septiembre 2019]. Disponible en: https://www.who.int/maternal_child_adolescent/topics/maternal/adolescent_pregnancy/es/

2. Organización Panamericana de la Salud (OPS). América Latina y el Caribe tienen la segunda tasa más alta de embarazo adolescente en el mundo [Internet]. OPS. 2018. [actualizado marzo 2020; consultado Septiembre 2019] Disponible en: https://www.paho.org/chi/index.php?option=com_content\&view=article\&id=996:america-latina-y-elcaribe-tienen-la-segunda-tasa-mas-alta-de-embarazo-adolescente-en-el-mundo\&ltemid $=1005$

3. Organización para la Cooperación y el Desarrollo Económico (OCDE). Situación de la Salud Sexual y Reproductiva de las entidades Federativas [Internet]. OCDE. 2017. [actualizado marzo 2020; consultado Septiembre 2019]. Disponible en: https://www.gob.mx/cms/uploads/attachment/file/236809/11_Guanajuato_web.pdf

4. Instituto Nacional de Estadística y Geografía (INEGI). Estadísticas Puebla [Internet]. INEGI. 2017. [actualizado marzo 2020; consultado Septiembre 2019]. Disponible en: https://www.diariocambio.com.mx/2018/especial/item/33480-puebla-octavo-lugar-con-mas-

embarazos-adolescentes-25-mil-casos-durante-2017

5. Secretaría de Salud (SS). Embarazo en Puebla [Internet]. SS. 2016. [actualizado marzo 2020; consultado Septiembre 2019]. Disponible en: https://www.milenio.com/estados/registran-puebla-94-embarazosmenores-15-anos-1

6. Norma Oficial Mexicana NOM-047-SSA2-2015, Para la atención a la salud del grupo etario de 10 a 19 años de edad. DOF Secretaria de Salud [Internet]. [consultado Junio 2020]. Disponible en: http://evaluacion.ssm.gob.mx/pdf/normateca/NOM-047-SSA2-2015.pdf

7. Consejo Nacional de Población. Estrategia Nacional para la Prevención del Embarazo en Adolescentes (ENAPEA). [Internet]. 2018 [consultado Junio 2020]. Disponible en: https://www.gob.mx/conapo/documentos/documento-oficial-de-la-estrategia

8. Loredo A, Vargas C, Casas M, González C, Gutiérrez L. Embarazo adolescente: sus causas y repercusiones en la diada. Rev Med Inst Mex Seguro Soc [Internet]. 2017 [consultado Septiembre 2019]; 55(2): 223-9. Disponible en: http://revistamedica.imss.gob.mx/editorial/index.php/revista_medica/article/viewFile/1379/1982

9. Chacón O, Cortes A, Álvarez G, Sotonavarro G. Embarazo en la adolescencia, su repercusión familiar y en la sociedad. Rev Cubana Obstet Ginecol [Internet]. 2015 [consultado Septiembre 2019]; 41(1): 50-8. Disponible en: http://scielo.sld.cu/pdf/gin/v41n1/gin06115.pdf 
10. Mora C, Hernández V. Embarazo en la adolescencia: cómo ocurre en la sociedad actual. Perinatol Reprod Hum [Internet]. 2015 [consultado Septiembre 2019]; 29(2): 76-82. Disponible en: https://doi.org/10.1016/j.rprh.2015.05.004

11. Mendoza T, Claros B, Peñaranda O. Actividad sexual temprana y embarazo en la adolescencia: estado del arte. Rev. chil. obstet. Ginecol [Internet]. 2016 [consultado Septiembre 2019]; 81(3): 243-53. Disponible en: http://dx.doi.org/10.4067/S0717-75262016000300012

12. Chamorro V, Padilla L, Gómez L, Quintero S. Percepción del estado de salud y comportamiento sexual y reproductivo en adolescentes embarazadas. Rev Enferm IMSS [Internet]. 2016 [consultado Septiembre 2019]; 24(1): 45-50. Disponible en: https://www.medigraphic.com/pdfs/enfermeriaimss/eim2016/eim161h.pdf

13. Estrada F, Campero L, Suárez L, Vara S, González C. Conocimientos sobre riesgo de embarazo y autoeficacia en hombres adolescentes: apoyo parental y factores escolares. Salud Pública Méx [Internet]. 2017 [consultado Septiembre 2019]; 59(5): 56-65. Disponible en: https://doi.org/10.21149/7959.

14. Figueroa L, Figueroa P. Conductas sexuales de riesgo en adolescentes desde el contexto cubano. Revista de Ciencias Médicas de Pinar del Río [Internet]. 2017 [consultado Septiembre 2019]; 21(2):14351. Disponible en: http://scielo.sld.cu/scielo.php?script=sci_arttext\&pid=S1561-31942017000200020

15. Álvarez N, Grande G, Linares A, Ojeda A. Análisis del embarazo adolescente: miradas cualitativas a los casos de Bucaramanga y Jaén. Matronas Prof [Internet]. 2017 [consultado Septiembre 2019]; 18(2): 51-59. Disponible en: https://www.federacion-matronas.org/matronas-profesion/sumarios/analisis-delembarazo-adolescente-miradas-cualitativas-a-los-casos-de-bucaramanga-y-jaen/

16. Mora, M. La teórica de las representaciones sociales de Serge Moscovici. Athenea digital [Internet]. 2002 [consultado Junio 2020]; 2: 1-24. Disponible en: https://doi.org/10.5565/rev/athenead/v1n2.55

17. Rivadeneira R. Comprensión teórica y proceso metodológico de la investigación cualitativa. In Crescendo (Chimbote) [Internet]. 2015 [consultado Septiembre 2019]; 6 (2): 169-83. Disponible en: https://doi.org/10.21895/incres.2015.v6n2.16

18. Vaismoradi M, Turunen H, Bondas T. Qualitative descriptive study. Nurs Health Sci [Internet]. 2013 [consultado Septiembre 2019]; 15 (3): 398-405. Disponible en: https://www.ncbi.nlm.nih.gov/pubmed/23480423

19. Hernández R, Fernández C, Baptista P. Metodología de la investigación. 6a ed. México: McGraw Hill Interamericana; 2014.

20. Berguera O, Fernandez de Sanmamed S, Pons V, Pujol R, Rodríguez A, Saura S. Escuchar, observar y comprender. Recuperando la narrativa en las ciencias de la salud. Aportaciones de la investigación cualitativa. [Internet]. 2014 [consultado Junio 2020]; Disponible en: https://saludcomunitaria.files.wordpress.com/2014/12/escucharobservarcomprender.pdf

21. Polit D, Hungler B. Investigación científica en ciencias de la salud. $6^{a}$ ed. México: McGraw- Hill Interamericana, 2000.

22. Braun V, Clarke V. Using thematic analysis in psychology. Nursing and Health Sciences [Internet]. 2013 [consultado Septiembre 2019]; 15: 398-405. Disponible en: https://www.researchgate.net/publication/267623311_Thematic_Analysis

23. Secretaría de Salud (SS). Reglamento de la Ley General de Salud en Materia de Investigación para la Salud. [Internet]. SS. 1987. [actualizado Marzo 2020; consultado Septiembre 2019]. Disponible en: http://www.salud.gob.mx/unidades/cdi/nom/compi/rlgsmis.html

24. Organización de las Naciones Unidas (ONU). Objetivos de Desarrollo Sostenible [Internet]. ONU. 2015 [actualizado Marzo 2020; consultado Septiembre 2019]. Disponible en: https://www.un.org/sustainabledevelopment/es/objetivos-de-desarrollo-sostenible/

25. Gómez I, Durán A. El acceso a métodos anticonceptivos en adolescentes de la Ciudad de México. Salud Pública Méx [Internet]. 2017 [consultado Septiembre 2019]; 59 (3): 236-47. Disponible en: http://dx.doi.org/10.21149/7891 
26. González A, Quintana L. La anticoncepción en adolescentes de Plaza de la Revolución: 1996-2011. CEDEM/Novedades en población [Internet]. 2015 [consultado Septiembre 2019]; 11(22): 90-7. Disponible en: http://scielo.sld.cu/scielo.php?script=sci_arttext\&pid=S1817-40782015000200010

27. Carrillo $S$, Manzanero R. Identificación de factores de riesgo y perspectivas de las adolescentes respecto al embarazo, sexualidad y anticoncepción. JONNPR [Internet]. 2018 [consultado Septiembre 2019]; 3(4): 268-78. Disponible en: https://doi.org/10.19230/jonnpr.2284

28. Mejía M, Laureano E, Gil H, Ortiz V, Blackaller A, Benítez M. Condiciones socioculturales y experiencia del embarazo en adolescentes de Jalisco, México: estudio cualitativo. Revista Colombiana de Obstetricia y Ginecología [Internet]. 2015 [consultado Septiembre 2019]; 66(4): 242-52. Disponible en: http://dx.doi.org/10.18597/rcog.291

29. Loredo A, Vargas C, Casas M, González C, Gutiérrez L. Embarazo adolescente: sus causas y repercusiones en la diada. Rev Med Inst Mex Seguro Soc [Internet]. 2017 [consultado Septiembre 2019]; 55(2): 223-29. Disponible en: http://revistamedica.imss.gob.mx/editorial/index.php/revista_medica/article/viewFile/1379/1982

30. Rojas B, Méndez V, Álvarez N. El papel de la familia en la normalización del embarazo a temprana edad. Revista Encuentros [Internet]. 2016 [consultado Septiembre 2019]; 14(1): 139-50. Disponible en: http://dx.doi.org/10.15665/re.v14i1.674

31. Mora C, Hernández V. Embarazo en la adolescencia. Revista de Ginecología y Obstetricia de México [Internet]. 2015 [consultado Septiembre 2019]; 83: 294- 01. Disponible en: https://ginecologiayobstetricia.org.mx/secciones/articulos-de-revision/embarazo-en-la-adolescencia/ 32. Quintero R, Rojas B. El embarazo a temprana edad, un análisis desde la perspectiva de madres adolescentes. Revista Virtual Universidad Católica del Norte [Internet]. 2015 [consultado Septiembre 2019]; 44(1): 222-37. Disponible en: https://www.redalyc.org/articulo.oa?id=194238608016

33. Ramos R, Borges C. Global self-esteem and maternal self-esteem in adolescent mothers. Rev Cubana Med Gen Integr [Internet]. 2016 [consultado Diciembre 2019]; 32(4): 1-11. Disponible en: http://scielo.sld.cu/scielo.php?script=sci_arttext\&pid=S0864-21252016000400004\&lng=es

34. Niño B, Ortiz R, Solano A, Amaya C, Serrano H. Concepciones del embarazo en adolescentes gestantes de la ciudad de Bucaramanga. Revista Cuidarte [Internet]. 2017 [consultado Diciembre 2019]; 8(3): 1875-76. Disponible en: http://dx.doi.org/10.15649/cuidarte.v8i3.448 